\title{
Simulation Study on the Potential of Braided Hose Failure in Internal Combustion Engines
}

\author{
S.Hassan ${ }^{1, *}$, S.Afdhal ${ }^{2}$, M.Shahrizal ${ }^{2}$ and A.Eleena ${ }^{1}$ \\ ${ }^{1}$ Department of Mechanical Engineering, Universiti Teknologi PETRONAS, 31750 Bandar Seri \\ Iskandar,Perak. \\ ${ }^{2}$ PETRONAS Carigali Sdn.Bhd, Kompleks Operasi PETRONAS, Kerteh, Terengganu, Malaysia.
}

\begin{abstract}
In this study, damaged braided hoses was obtained from the actual site to investigate the possible causes of failure in the internal combustion engines. The hoses were assembled with polytetrafluroethylene (PTFE) as the inner tube and externally braided outer tube corrosionresistant steel (CRES). The hose had undergone failures near the end of the fittings. The braided hose was measured and modelled using SOLIDWORKS CAD software. The ANSYS static structural stimulation was used to measure the stress distribution on the hose due to pressure applied with various bend shapes. The simulation was done by fixing the end of the pipe and exerting the working pressure inside the inner layer of PTFE. The results of the simulation have shown that the inner tube experienced high stresses near the fittings which is then aggravated with the bending. With cyclic pressure loading, the hose was unable to stand the high stresses near the fittings and therefore failed. Further analyses were done to the braided hose to investigate the other possible of failure using failure analysis method.
\end{abstract}

\section{Introduction}

A hose is defined as a piece of tubing that is commonly used to move liquids or gases from one point to another. In the industrial application, hoses need to withstand a high pressure and high temperature environment which hoses are made of metal. Stainless hoses are widely used in the industry due to high physical strength combined with light weight, as well as to prevent corrosion. The stainless steel hose also used when it is needed to absorb the heat or pressure-induced expansion of the pipe system for major equipment where the system is exposed to high external heat, corrosion or heavy rough handling, the metal hose are flexible and can withstand high pressure (Pierce \& Evans, 2012).

Generally, hoses are classified by types which are non-metallic and metallic. The nonmetallic hose is made of rubber tubing which are using the material of Polyvinyl chloride (PVC) (Spellman, 2008). In the industrial application, braided hoses are more preferred due to its high flexibility and at the same time to adapt the movements of the hose. Comparing to non braided corrugated hoses, braided hoses prevents longitudinal expansion of corrugated hose.

\footnotetext{
*Corresponding author: suhaimiha@utp.edu.my
} 
The application of the braided stainless steel hoses are in addition to take up misalignment or thermal expansion of the gas pressure during the operation of the turbine and generator. Vibration and noise are also absorbed from major equipment such as pumps, compressors and engines during running of the machines. The applications of stainless steel flexible connections particularly in automotive industry are commonly for fuel distribution such as connections to the lubricating oil system.

\section{Background of Study}

Failure of braided hose in the major equipment in the internal combustions engines such as diesel generator-set and gas turbine is one of the concern and major problem faced nowadays. The common area of failure is seen on the flexible connectors of the pipe because the flexible connectors are combined through welding which produced weak points of assembly. Most of an allowable pressure in the stainless steel hoses as claimed by the manufacturer is around $1500 \mathrm{psi}$. Therefore, the working pressure larger than that could lead further to the failure of the stainless steel pipe (Márquez et al, 2009).

In practical, the braided hose are placed as an external layer of stainless steel provided to the hose. According to Cho et al. (2013), flexible hoses comprising a wire spiral support embedded in a plastic pipe wall are frequently used as part of vacuum transfer or pneumatic conveying systems in a wide range of industries where powders are handled, processed or manufactured. However, the actual hose prototype is reinforced with stainless steel outer tube and polytetrafluroethylene (PTFE) inner tube protects the hose from chemical and corrosive gases. PTFE is an engineered fluropolymer; outstand to resist a broad temperature range.

PTFE polymer has a quality characteristic of polymer that is the best damping coefficient (Okularczyk, 2007). In addition, the stainless steel hose are good vibration and noise absorber, it more efficient with PTFE material as an inner tube.

Hachemi et al., 2011 mentioned that flexible stainless steel hose are used in many applications, for example to maximize the effect of vibration on piping and car engines. The hose will fail due to dynamic loads from gas and liquid pulsation. Hachemi added that small holes or crack are the initial point of failure for hoses. Therefore, the conditions of leak before break will occur most in prediction of damage. This is valuable as it enables the reduction of the failure consequences in a working environment. (Hachemi et al., 2011)

According to Pierce \& Evans (2012) flexible hose that did not meet the extreme operating conditions of the assembly might cause failures in industrial applications. The problem will not only cost from an operational and schedule stand point, but it also creates a significant safety risk. It is imperative that the mechanical characteristics that are display are well known and taken into consideration during the design of the assembly with the flex hoses being integral part in major engineering assemblies such as turbine and generator. In brake hoses, the combined effects of years of flexing, high pressure and exposure to the harsh environment at the lower chassis will eventually deteriorate the brake hoses (Baaser, 2007).

Marquez et al., 2009 mentioned the fracture in the wire braid could be a possible phenomenon of fatigue crack propagation in previous worn areas that was not detected earlier. The flexible hose connection was over pressured than the maximum allowable limit defined by the manufacturer. With the widely used materials hose of plastic (PE and PVC) for common hose, it is expected the polymer will age and eventually fail (Rostum, 2008).

A key factor of the presence of contaminants of hydrocarbon leads from the ignition point and following the path of flow are the reason of failure. The failure occurred on the cylinder end, near the connector or fittings of the braided stainless steel hose. There were small holes found near the connectors or fittings were caused by the thinning of the liner PTFE due to ignition. As shown in Fig. 1, the point of failure stated is most similar failure point when compared to the actual prototype of the braided hose failure (Royals et al, 1997). 


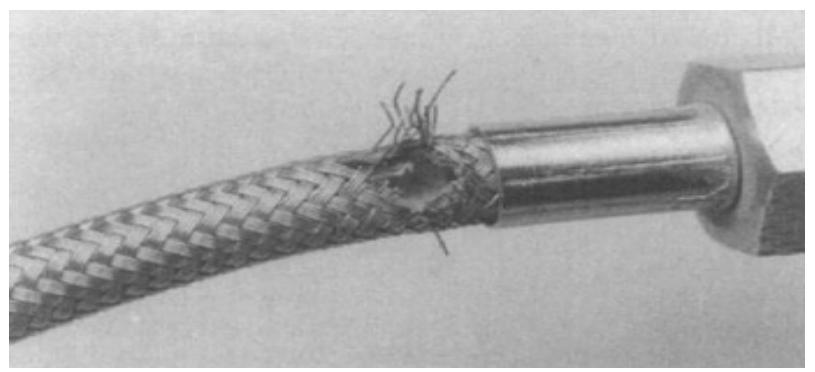

Fig. 1. Failed section of braided hose.

Based on literature study reviewed, the most common causes of failure in hydraulic hoses are the multi-plane bending that result in twisting of its wire reinforcement. The service life of a high-pressure hydraulic hose is reduced as much as $70 \%$ for twist of five degrees and a seven degree twist can result in a 90\% reduction in service life. Multi-plane bending occurs from inadequate or unsecure clamping where the hose is subjected to vibration of machine or actuator movement (Voirin, 2011). The hydraulic hose are recommended to be segmented to prevent multi-plane bending as shown in Fig.2.

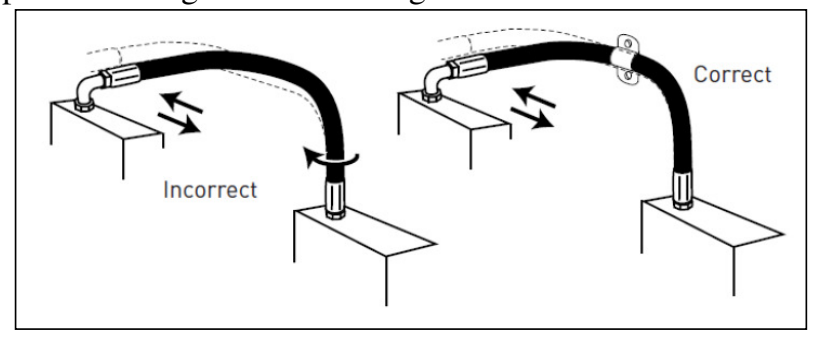

Fig. 2. Recommended installation of braided hose.

Addition to the multi-bending, a failure due to hose over pressure would damage the braid and result in a longitudinal split in the inner core of the hose. The hoses inner core, causes the stress in the object to be non-uniform, where the part of the object on the outside of the bend is in tension, and the inside of the bend is in compression causes by the bending of the cylindrical object. This would result in the outside of the bend being the most prone to failure where the area of the highest normal and shear stresses. Failure would occur starting directly on top of the outside of the bend, somewhere near the connector (Voirin, 2011).

In order to analyze the braided hose failure, the use of Finite Element Analysis (FEA) is a common nowadays. FEA is a computerized method for predicting how a product reacts to real-world forces, vibration, heat, fluid flow, and other physical effects. Finite element analysis shows whether a product will break, wear out, or work the way it was designed.

According to Lee et al. (2011) using FEA, swaging part of the hose needs be analyzed because it often offers the reason why failure modes occur, like leakage as stress concentration and where in connecting part between metal and rubber part under high pressure conditions. Additionally, according to Pierce \& Evans (2012), due to elastic strain the braid retracted but not retract to its original position due to plastic deformation.

However, with the pressure cycle testing, the pressure needed to be much greater than maximum pressure before the elongation of the hose would continue. Finite Element model helped in better understanding of what could have caused the premature failure of the hose. The Finite Element model can be done by setting different distance from the leak hole of the pipe. This has a different corresponding frequency value to the vibration maximum amplitude. Each node are placed to indicate the different distances and in 0 degree angle to the circumferential direction of the leak hole (Liang, 2013). 
Zhang \& Xu (2013) suggests the method of Finite Element Analysis can be built up of identical unit cells by yarn configuration in three regions and three axis of $\mathrm{x}$, $\mathrm{y}$ and $\mathrm{z}$. With an appropriate boundary conditions, the mechanical response can be simulated and deformation and stress distribution of unit cell models can be presented and the effects the braiding angle are investigated in detail. The curved yarn path in the exterior surface and corner unit cell models are considered as one straight line for the sake of simplicity.

Therefore, most of the common causes of failure in hydraulic hoses are the multi-plane bending that result in twisting of its wire reinforcement. The service life of a high-pressure hydraulic hose is reduced as much as $70 \%$ for twist of five degrees and a seven degree twist can result in a $90 \%$ reduction in service life. Multi-plane bending occurs from inadequate or unsecure clamping where the hose is subjected to vibration of machine or actuator movement (Voirin, 2011).

\section{Methodology}

In this study, the actual sample of braided hose was used with inner tube of polytetrafluroethylene (PTFE) and externally braided outer tube corrosion-resistant steel (CRES), taken from the plant. The sample of braided hose was divided into section to improve the analysis of study as shown in Fig. 3. This is done in order to identify the specific section of failure that occurs on the hose assembly.

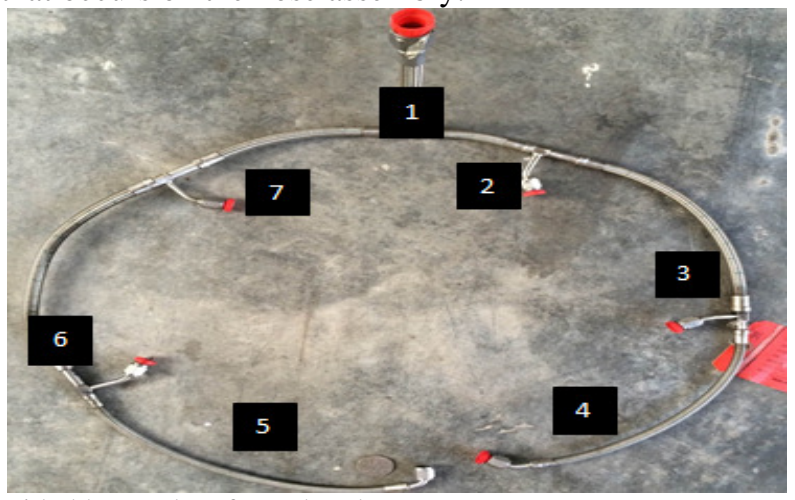

Fig. 3. Sample of braided hose taken from the plant.

The failure parts of the hose occur have been identified in sections 3 and 6 , respectively. However, hose 3 has minimal damage and the hose is not elongated from its original diameter. In addition, section of hose 6 , were undergone elongation and burned. Hose section 3 and 6 are assumed to be same initial length and diameter without fittings, as shown in Fig. 4 and Fig.5, respectively.

The hose are cut into cross section to identify the fitting details of the hose. The end of the pipe are clamped by fittings $15.27 \mathrm{~mm}$ away from visible hose. The length of the pipe is $220 \mathrm{~mm}$ for both stainless steel and PTFE excluding the fitting.

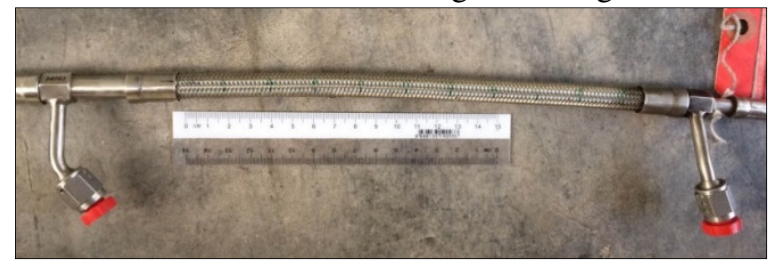

Fig. 4. Sample of braided hose taken from the plant. 


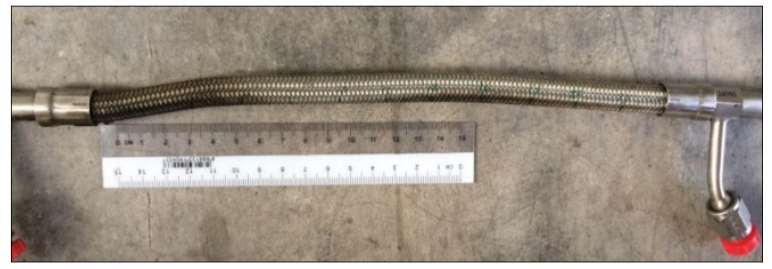

Fig. 5. Section 6 of the braided hose sample.

The hose was cut into cross section to identify the fitting details of the hose as shown in Fig. 6. The end of the pipe are clamped between two layer fittings $15.27 \mathrm{~mm}$ away from visible hose. The length of the pipe is $220 \mathrm{~mm}$ for both stainless steel and PTFE excluding the fitting. Both the hose outer and inner diameter are measured using vernier caliper in the lab.

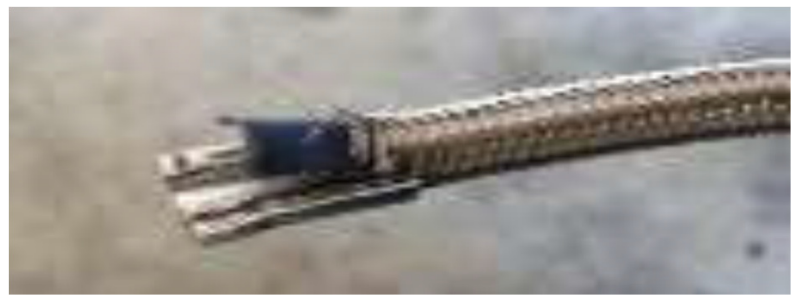

Fig. 6. Cross section of the braided hose sample.

The sample of braided hose initially was modeled using Solidworks software and various in shapes; horizontal, $\mathrm{U}$ shape, $\mathrm{L}$ shape and $\mathrm{S}$ shape. Then followed by simulation work using ANSYS static structural. The physical properties and static structure setup were set up for each shape of model. with the physical properties of the material inserted in the simulation is shown in Table 1.

In the simulation work, pressure of $10.342 \mathrm{MPa}$ was applied on the inside of the tube (inner layer PTFE), and the results are shown in term of Von Mises stresses and total deformation of the braided hose.

Table 1. Physical Properties of CRES and PTFE.

\begin{tabular}{|l|c|l|l|}
\hline \multicolumn{1}{|c|}{ Parameters } & Unit & \multicolumn{1}{c|}{ CRES } & \multicolumn{1}{c|}{ PTFE } \\
\hline Density & $\mathrm{kg} / \mathrm{m}^{3}$ & 7750 & 2200 \\
\hline Young's Modulus & $\mathrm{Pa}$ & $1.9 \mathrm{E}+11$ & $5 \mathrm{E}+08$ \\
\hline Poisson's Ratio & $\mathrm{Pa}$ & 0.31 & 0.46 \\
\hline Bulk Modulus & $\mathrm{Pa}$ & $1.693 \mathrm{E}+11$ & $\begin{array}{l}2.0833 \mathrm{E}+1 \\
0\end{array}$ \\
\hline Shear Modulus & $\mathrm{Pa}$ & $7.3664 \mathrm{E}+10$ & $\begin{array}{l}1.7123 \mathrm{E}+0 \\
9\end{array}$ \\
\hline $\begin{array}{l}\text { Tensile Yield } \\
\text { Strength }\end{array}$ & $\mathrm{Pa}$ & $2.07 \mathrm{E}+08$ & $2.3 \mathrm{E}+07$ \\
\hline
\end{tabular}

Von Mises stress is widely used to check whether the geometry will withstand a given load condition. The concept of Von Mises stress arises from the distortion energy failure theory which is distortion by volume and angular shape of the design. The hose will fail, if the maximum value of Von Mises stress induced in the material is more than strength of the material. The yield strength for stainless steel and polytetrafluroethylene (PTFE) is $207 \mathrm{MPa}$ and $23 \mathrm{MPa}$, respectively.

Total deformation occurs due to the braided hose expansion and elongation when the pressure is exerted to it. This deformation is due to the properties of material which involves 
elastic and plastic deformation. The structure is forced to expand and compress due to the pressure exerted, then the maximum deformation are observed.

Therefore, the results obtained were based on the ANSYS static structural simulation done on the actual model of braided hose. The braided hose failures on hose section 3 and 6 are predicted through stress distribution of Von Mises equivalent stress and total deformation.

\section{Results and Discussion}

The braided hose failures were predicted based on ANSYS static structural simulation to determine the Von Mises stress and total deformation of braided hose for PTFE and full body (FB) at different hose orientation.

\subsection{Horizontal position}

The horizontal hose simulation results for Von Mises stress with PTFE and FB are shown in Fig. 7.

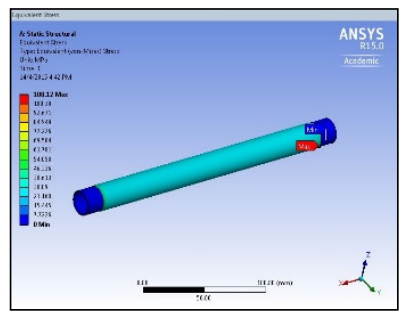

(a)

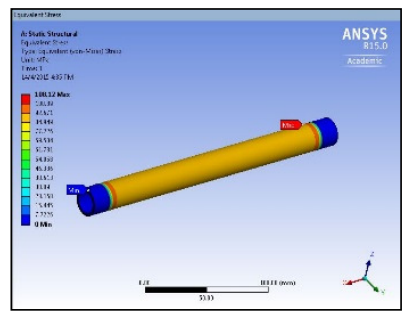

(b)

Fig. 7. Von Mises stress on U-shape with PTFE (a) and FB (b).

The maximum Von Mises stress is $108.12 \mathrm{MPa}$ and the minimum is $0 \mathrm{MPa}$. According to the yield strength of the stainless steel, the design is safe. The Von Mises stress for the horizontal hose is less than the yield strength of stainless steel which is $207 \mathrm{MPa}$. By installation of horizontal position, there was no stress failure detected. The maximum stresses are mostly detected near the fittings of the hose. Total deformation as in Fig. 8 shows the maximum point at $0.5572 \mathrm{~mm}$ where both the material can withstand the deformation of plastic and elastic.

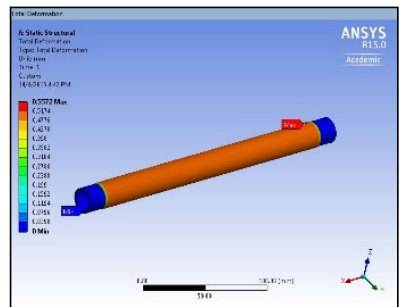

(a)

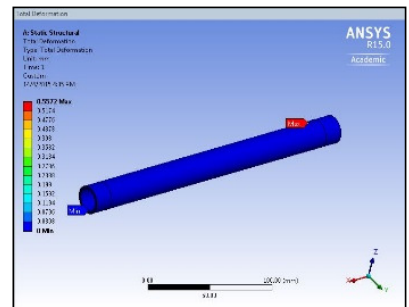

(b)

Fig. 8. Total deformation on horizontal with PTFE (a) and FB (b).

\subsection{U-shape position}

Figure 9 shows the U-shape position of PTFE and FB. The maximum Von Mises stress is 137.32 MPa and the minimum is $0 \mathrm{MPa}$. According to the yield strength of the stainless steel, the design is safe. The Von Mises stress for the horizontal hose is again less than the yield strength of stainless steel which is $207 \mathrm{MPa}$. 


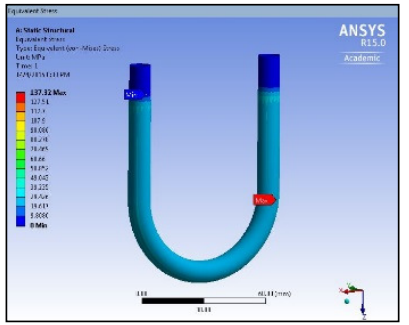

(a)

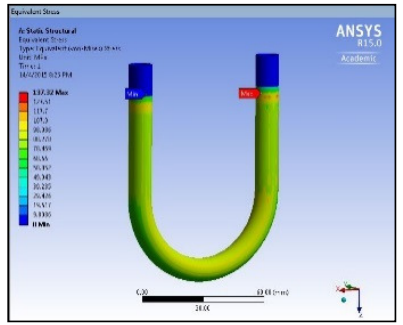

(b)

Fig. 9. Von Mises stress on U-shape with PTFE (a) and FB (b).

The total deformation in Fig. 10 shows that the maximum total deformation is 0.30033 $\mathrm{mm}$ for $\mathrm{U}$ shape hose position which is less than the maximum for horizontal hose position. This indicates, horizontal position have a higher chance of deforming. According to the observation of the results for the layer of stainless steel and PTFE, the total deformation is maximum again near the fittings of the hose. Due to the pressure exerted inside the PTFE layer and fixed support at the end of the hose, the deformation is concentrated near the fittings. However, the PTFE layer did not deformed beyond the stainless steel layer as predicted.

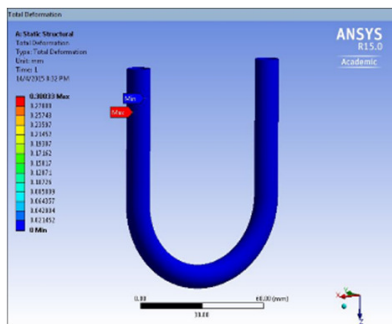

(a)

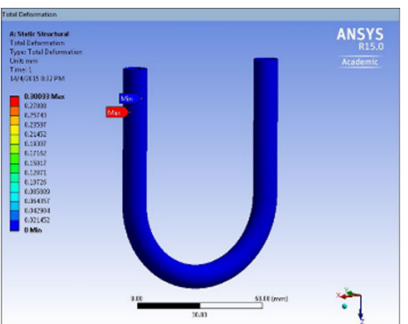

(b)

Fig. 10. Total deformation on U-shape with PTFE (a) and FB (b).

\subsection{L-shape position}

Based on Fig. 11, the maximum Von Mises stress is $88.432 \mathrm{MPa}$ and the minimum is $0 \mathrm{MPa}$. $\mathrm{L}$ shape position indicates that it would produce smaller Von Mises Stress compared to horizontal hose and $\mathrm{U}$ shape hose installation. By installation of $\mathrm{L}$ shape position, there was no stress failure detected. The maximum stresses are mostly detected near the fittings with and without the stainless steel material.

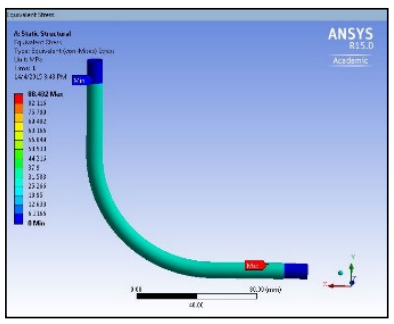

(a)

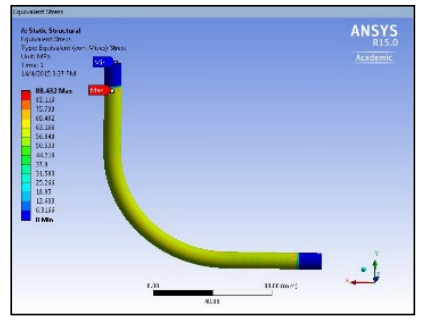

(b)

Fig. 11. Total deformation on L-shape with PTFE (a) and FB (b). 


\subsection{S-shape position}

The maximum Von Mises stress for S-shape is $159.8 \mathrm{MPa}$ and the minimum is $0 \mathrm{MPa}$, as shown in Fig. 12. S shape position indicates that it would produce much larger Von Mises Stress compared to the other hose installation. By installation of $S$ shape position, there was no stress failure detected but near to failure. The maximum stresses are mostly detected near the fittings of the hose. The full body of the $\mathrm{S}$ shape hose indicates maximum Von Mises diverted the Von Mises stress away from the fittings.

Figure 13 shows the maximum total deformation is $0.28982 \mathrm{~mm}$ for $\mathrm{S}$ shape hose position which is smallest deformation compared to other installation position. This further proves, horizontal position have a highest total deformation. According to the observation of the results for the layer of stainless steel and PTFE, the total deformation is maximum near the fittings. Due to the pressure exerted inside the PTFE layer and fixed support at the end of the hose, the deformation is concentrated near the fittings. However, the PTFE layer did not deformed beyond the stainless steel layer as predicted.

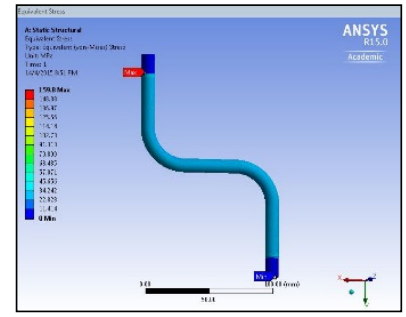

(a)

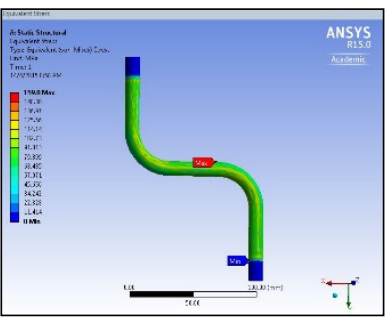

(b)

Fig. 12. Von Mises stress on S-shape with PTFE (a) and FB (b).

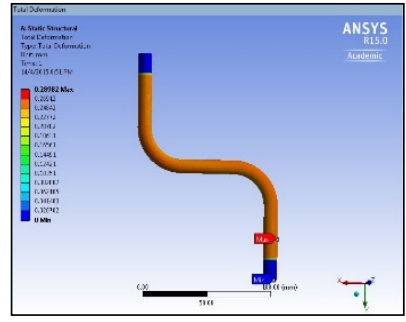

(a)

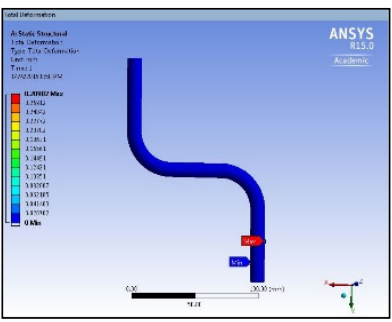

(b)

Fig. 13. Von Mises stress on S-shape with PTFE (a) and FB (b).

\section{Conclusion}

Braided hose leakage in equipment will inflict significant cost in downtime and material wastage. It is crucial to investigate factors to improve the braided hose reliability and mechanism to detect the potential leakage. From the results, the highest maximum Von Mises stress is $159.8 \mathrm{MPa}$ for $\mathrm{S}$ shaped hose. The lowest maximum Von Mises stress is L shaped hose which is $88.432 \mathrm{MPa}$. The total deformation results show that the horizontal position has the highest maximum deformation of $0.5572 \mathrm{~mm}$ and lowest total deformation is for $\mathrm{S}$ shape hose with value $0.28982 \mathrm{~mm}$. The deformation has minimal effect on the braided hose failure. However, both Von Mises stress and total deformation have confirmed that the maximum values of stress and deformation occurs near fittings. The best shape for installation is horizontal hose position for minimum stress on braided hose. To prevent the hose from multi-plane bending, the hoses should be installed with adapters. A hydraulic hose subjected to machine or actuator movement should be routed properly with bracket or installed on the wall to prevent failure of the hose near point of fittings. 
The authors would like to express sincere appreciation to PETRONAS Carigali Kerteh for the financial support for this project, also to the Department of Mechanical Engineering Universiti Teknologi PETRONAS.

\section{References}

1. W. Okularczyk, Arch. Civ. Mech. Eng., 7, 167 (2007)

2. H. Hachemi, H., Kebir, H., Roelandt, J. M., \& Wintrebert, E, Mater. Design, 32, 1957 (2011).

3. S. Pierce \& Evans, J.L., Eng. Fail. Anal., 22, 11 (2012).

4. H. Baaser, Comp. Mater. Sci., 39, 113 (2007).

5. A. Márquez, A., Fazzini, P. G., \& Otegui, J. L., Eng. Fail. Anal., 16, 1912 (2009).

6. J. Rostum, Statistical Modelling of Pipe Failures in Water Networks. Department of Hydraulic and Environmental Engineering. (2008).

7. G. Royals, W. T., Chou, T. C., \& Steinberg, T. A., Flammability and Sensitivity of Materials in Oxygen Enriched Atmospheres. American Society of Mechanical Engineering (ASME) (1997).

8. E. Voirin, E., COUPP-60 Hydraulic Hose Failure Analysis. Mechanical Department Engineering Note. Fermilab. (2011).

9. R. Spellman, Handbook of Water and Wastewater Treatment Plant Operations (Vol. Second Edition). CRC Press, (2008).

10. J. Cho, Jee, Y.B., Kim, W.J., Han, S.R., Lee, S.B., Compos. Part B: Eng., 53, 112 (2013).

11. C. Zhang, C. \& X. Xu, Compos. Struct., 98, 130 (2013).

12. W. Liang, W., Zhang, L., Xu, Q., Yan, C., Eng. Fail. Anal., 31, 1 (2013).

13. G. Lee, G.-C., Kim, H.-E., Park, J.-W., Jin, H.-L., Lee, Y.-S., \& Kim, J.-H, Int. J. Precis. Eng. Man., 12, 537 (2011). 